\title{
Optomechanical Coupling Active Control for Improving Beam Pointing Accuracy of the Spatial Filter in PW Laser Facility
}

\author{
Wenhui Cui ${ }^{1,2, *}{ }^{\mathbb{D}}$, Jianqiang Zhu ${ }^{1}$, Zhigang Liu ${ }^{1}{ }^{\circledR}$, Quantang Fan ${ }^{1}$, Zhaoyang Jiao ${ }^{1}{ }^{\circledR}$, Junyong Zhang ${ }^{1}$, \\ Weiheng Lin ${ }^{1,2}$ and Ziming Dong ${ }^{1}$ \\ 1 Joint Laboratory on High Power Laser and Physics, Shanghai Institute of Optics and Fine Mechanics, \\ Chinese Academy of Sciences, Shanghai 201800, China; jqzhu@siom.ac.cn (J.Z.); 1zg_siom@siom.ac.cn (Z.L.); \\ Qtfan@siom.ac.cn (Q.F.); zhyjiao@siom.ac.cn (Z.J.); zhangjy829@siom.ac.cn (J.Z.); whlin@siom.ac.cn (W.L.); \\ dongziming@shu.edu.cn (Z.D.) \\ 2 University of Chinese Academy of Sciences, Beijing 100049, China \\ * Correspondence: whcui@siom.ac.cn
}

Citation: Cui, W.; Zhu, J.; Liu, Z.; Fan, Q.; Jiao, Z.; Zhang, J.; Lin, W.; Dong, Z. Optomechanical Coupling Active Control for Improving Beam Pointing Accuracy of the Spatial Filter in PW Laser Facility. Appl. Sci. 2021,

11, 5017. https://doi.org/ 10.3390/app11115017

Academic Editor: David Andrews

Received: 27 April 2021

Accepted: 25 May 2021

Published: 28 May 2021

Publisher's Note: MDPI stays neutral with regard to jurisdictional claims in published maps and institutional affiliations.

Copyright: (C) 2021 by the authors Licensee MDPI, Basel, Switzerland. This article is an open access article distributed under the terms and conditions of the Creative Commons Attribution (CC BY) license (https:/ / creativecommons.org/licenses/by/ $4.0 /)$.

\begin{abstract}
To improve the beam pointing accuracy of PW laser facility and reduce the optical axis deviation caused by the deflection amplitude response deviation of the confocal lens of spatial filter for microvibration action, an Optomechanical coupling vibration active control theory is proposed to make the peak value of output optical angle response lower than the pointing threshold value by $0.2 \mu \mathrm{rad}$. To establish an Optomechanical coupling vibration active control system, the active control parameters are introduced into the beam transmission matrix of the Optomechanical coupling system. The active control parameters and the peak value of the output light angle response are linked point to point. The algorithm flow of the active control system is designed, the control rules are established, and the control effect is verified. The results show that the peak value of the output optical angle response of the spatial filter decreases by $98.13 \%$, and the attenuation is nearly two orders of magnitude after the active control, which effectively improves the convergence accuracy of the beam pointing of the spatial filter of the PW laser facility, and realizes the beam pointing control under the broadband excitation, and the control result is consistent with the expectation.
\end{abstract}

Keywords: Optomechanical coupling; PW laser facility; active control; convergence accuracy

\section{Introduction}

To meet the requirements of physical experiments, all laser beams are required to be accurately positioned on the target. The mainstream ICF facilities, such as the first generation of Shiva facility [1], NIF facility [2], and Astra-Gemini@CLF facility [3], LMJ facility [4], SGII facility, SGII-Up facility [5-8], and SGIII facility [9] in China all have high requirements for the pointing accuracy of the laser beam. In recent years, many countries are racing to build PW laser facilities [10]. PW laser can create unprecedented comprehensive extreme physical conditions, such as super strong electromagnetic field, ultrahigh energy density, and ultrafast time scale in the laboratory. It has great application value in laser acceleration, laser fusion, attosecond science, astrophysics, nuclear physics, high energy physics, atomic and molecular physics, nuclear medicine, and other fields. It is one of the leading edges of international laser technology competition. PW laser facility has a small focal spot and high energy. High energy requires the size of the focal spot, while the size of the focal spot has requirements for pointing stability, and the PW facility has requirements for pointing stability [11,12]. LLNL in literature [13] proposed an interference adaptive optical system for high power laser beam correction in ARC. The directivity stability requirement is increased from $\pm 75 \mu \mathrm{m}$ to $\pm 10 \mu \mathrm{m}$, which sets higher requirements for the positioning error.PW laser facility is a large ultraprecision optical system, which has a long beam propagation distance and involves many optical elements. The microdisturbance of optical elements in the optical system will affect the 
beam pointing stability and beam focusing performance. The error of beam pointing stability can be divided into static error and dynamic error. The static error, such as assembly error, collimation error, and beam pointing error caused by slow drift, is relatively easier to adjust and control, while the dynamic error, such as structural vibration and thermal disturbance, is random disturbance and difficult to control.

In recent years, the problem of beam jitter suppression of optical facilities has attracted more and more attention from scientific research teams, and the vibration of the optical system has become frontier research. Mahdieh [14] and Gao [15] have studied the influence of the optical system misalignment on beam quality under vibration. Wang [16], and others proposed a beam correction principle by calibrating the beam pointing path and beam position path, which uses the relationship between the rotation angle and the PSDs signal to correct the beam drift. Chang [17] proposed an interference classification method based on LSTM and GRU to select appropriate control parameters to achieve the purpose of beam pointing stability control. Recently, a cavity free Optomechanical coupling scheme has been experimentally implemented in III-V semiconductor micromachining system, which provides enlightenment for the development of functional integrated Optomechanical devices [18]. In order to suppress the beam jitter, we need to start from the dynamics, control theory, and optical field, to find the law and reason of the optical system maladjustment, and design the appropriate controller. There are three kinds of beam pointing control methods: Precision design of the optical structure, vibration control of the optical mechanical structure, and beam stabilization technology.

The precision design of optical structure only focuses on the optical field, and improves the antivibration performance of the optical system by means of more precise optical element processing and adjustment [19]. In this way, the cost of it is very high. And it will be powerless in case of complex environmental interference. Beam stabilization technology can improve the misaligned beam trajectory and quality, which focuses on the adjustment of the beam itself, and is also a good way to improve the beam quality [13]. The vibration control of the Optomechanical structure can improve and eliminate the beam misalignment by eliminating the vibration of Optomechanical structure. This method focuses on the source of laser beam misalignment and focuses on stabilizing the mechanical elements in the optical system. As early as 1953, American astronomer Babcock [20] began to solve the problem of beam jitter of optical devices by means of mechanical vibration, and achieved a good control effect. Mcmickell, M.B. [21] and others have developed a vibration isolation device, which has been used in optical loading satellite experiments. Lee [22] established a complete frame disturbance and vibration isolation optical system model, and studied the control technology of disturbance caused by reaction flywheel. In the error analysis of the National Ignition Facility (NIF), the dynamic error accounts for $92 \%$ of the total error, while the static error only accounts for $8 \%$, and $60 \%$ of the dynamic error comes from structural vibration [23]. Therefore, it is very important to reduce the structural response amplitude of the optical facility and subsystems by solving the structural vibration error caused by random interference.

For large laser facilities, beam stabilization technology and optical structure precision design were used to improve the pointing accuracy in the past. The authors of [9] studied the structural stability control strategy of the SGIII laser facility, and proposed the stability index allocation method to obtain the structural stability design parameters of laboratory platform and optical components, which verified the feasibility of the strategy. The interference of the optical system caused by structural vibration, thermal gradient, or acoustic vibration will reduce the aiming accuracy and success rate [24]. The authors of [24] analyze the structural vibration of the National Ignition Facility (NIF) by using the finite element model. The authors of [25] discuss the stability of the mirror support system in the NIF. Yang [26] proposed a scheme to reduce the effect of beam direction jitter in a laser triangular displacement probe composed of a collimated red laser, a laser pointing control device, a receiving lens, and a charge coupled device. Qin [27] proposed that the tracking control of beam pointing is based on the Jacobian matrix, which can carry out effective self- 
calibration through active motion. The automatic collimation beam stabilization technology is effective for high firing rate shooting, but it is meaningless for low firing rate shooting. The space for optimization and improvement after the completion of structural design is limited. The above two methods may not meet the requirements of beam positioning index. To solve the problem of beam pointing stability, the Optomechanical coupling active control technology can be used to actively reduce the structural response to achieve the corresponding index requirements.

The authors of [28] analyze the beam positioning stability of a spatial filter caused by microvibration. Because most of the optical components of a large optical system are installed on the truss, which can be disturbed by the environment, it causes the incident beam to change with time, and the beam moves back and forth in the target position. As the spatial filter is rigidly connected with the support truss, the vibration is transmitted from the support truss to the top of the truss and then transmitted to the spatial filter. By controlling the vibration of the truss in the highly sensitive direction, the vibration response of the lens system in the highly sensitive direction can be suppressed.

At present, the research on the coupling of vibration active control technology into Optomechanical structure has not been put forward. The existing research has only analyzed the beam pointing stability by assuming that the structural vibration is known [29], but has not analyzed the structural transmission characteristics of the structure under excitation. Due to the high precision requirement of vibration control in the field of precision Optomechanical, such as PW laser facility, the active vibration control technology, should be coupled into the Optomechanical system when modeling.

Micro vibration is the main factor that affects the optical axis deviation of the system. In order to make the spot converge in the ideal position, this paper takes PW laser facility space filter Optomechanical system as the research object, analyzes the beam imbalance principle of the Optomechanical system, designs the split support scheme of long-distance optical spatial filter, identifies the modal parameters through a vibration test, constructs the Optomechanical structure equivalent model, and further constructs the Optomechanical coupled vibration active control system. The active control parameters are introduced into the beam transmission matrix of the Optomechanical coupling system, and the beam transmission model is modified. Through the first stage coupling of active control parameters and structural response and the second stage transfer of structural response and beam direction, the point-to-point link between active control parameters and beam pointing is realized. The active controller and voice coil motor active actuator are designed. The formula of output light angle response peak value is deduced. The influence of excitation amplitude, frequency, and phase on the peak value of output optical angle response is analyzed. The main factors affecting the beam pointing are found. The algorithm flow of the active control system is designed. The control rules are established, and the control effect is verified. The convergence characteristics of the beam pointing accuracy of the active control system compared with the noncontrol system is studied. It can be extended to PW facility full link space filter and similar system, which has certain engineering value.

\section{Principles and Methods}

\subsection{Beam Misalignment Principle of the Optomechanical System}

The misalignment principle diagram of the spatial filter and its supporting truss coupling Optomechanical system is shown in Figure 1. Figure 1a shows the torsion posture of the integrated support ANSYS. When the response of the Optomechanical structure is inconsistent under the excitation or the rigid center of the supporting structure does not coincide with the mass center, the optical axis of the spatial filter under the integrated support will deviate. Figure $1 \mathrm{~b}$ shows the posture of the split support torsion pendulum. When the lens offset of the two support trusses in the $x$ direction is different, the optical axis of the spatial filter under the split support will also deviate. 


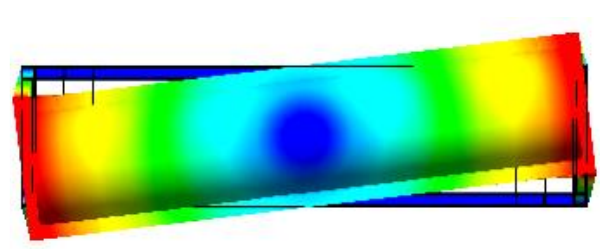

(a)

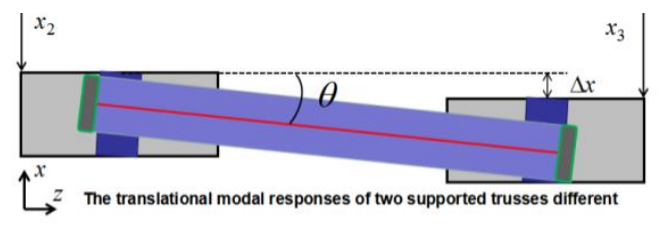

(b)

Figure 1. Misalignment principle diagram of spatial filter and its support truss coupled Optomechanical system: (a) Integrated support ANSYS torsion pendulum posture; (b) split support torsion pendulum posture.

To obtain the minimum amplitude of the output light angle, the ideal situation is to install the spatial filter on the integrated support truss, as shown in Figure 1a. When the spatial filter is long, torsion will also appear when it is placed on the integrated support truss. It is also reasonable to install the spatial filter on two identical split support trusses, as shown in Figure 1b, considering the factors of duty cycle and rationality of experimental layout. When installing a long optical path spatial filter in the laboratory, two identical trusses are usually used to support a confocal lens spatial filter system.

When the excitation amplitude, frequency, or phases of the two support trusses are different, the translational modal response of the space filter support trusses in $x$ direction is different. The system optical axis deviates from the static optical axis, and the system optical axis and the static optical axis are distributed at $\theta$ angle, which will cause the beam transmission maladjustment. Therefore, it is significant to study and analyze the convergence of beam pointing offset response caused by different amplitude, frequency, or phase excitation when the spatial filter system is placed on two identical support trusses.

\subsection{Modal Parameters Identification of the Optomechanical Coupling System}

To identify the modal parameters of the spatial filter Optomechanical coupling system, the vibration test was carried out in the experimental hall. Beijing East Institute vibration test analyzer (DASP) was used. The sensor is a high-sensitivity piezoelectric acceleration sensor (model is 393B05) from PCB company of USA, with sensitivity $\mathrm{K}_{\mathrm{CH}}=10 \mathrm{~V} / \mathrm{g}$. The measurement frequency range is $0.5-450 \mathrm{~Hz}$. The sampling frequency is $200 \mathrm{~Hz}$. It can be seen from Figure 2 that the frequency of the vibration response signal in the $\mathrm{Y}$ direction of the spatial filter support truss of the PW laser device is mainly within $20 \mathrm{~Hz}$, and the frequency of the vibration response signal in $X$ and $Z$ directions is about $3.18 \mathrm{~Hz}$, indicating that the energy of the response signal in each direction of the support truss is mainly concentrated near these frequencies. The modal parameters of the structure in all directions can be identified according to the actual working conditions.

This paper focuses on the $x$ direction modal parameters, which have a great influence on the beam pointing. It can be seen from Figure 2 that the $x$ direction modal frequency is concentrated around $3.18 \mathrm{~Hz}$ (i.e., $20 \mathrm{rad} / \mathrm{s}$ ). Refer to the actual working conditions to determine the modal parameters of the optical mechanical structure, as shown in Table 1.

According to the response amplitude frequency and phase frequency curve of Figure 3 Optomechanical structure, the natural frequency of the uncontrolled spatial filter is $20 \mathrm{rad} / \mathrm{s}$ and the transmission rate is 15.7. The parameters are used as the reference index for the subsequent research. 

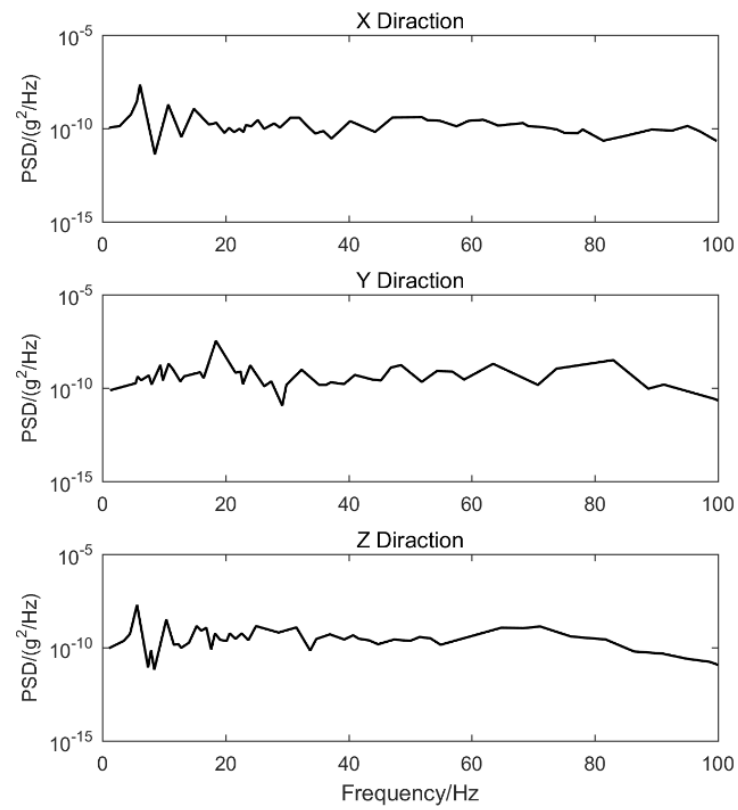

Figure 2. Triaxial vibration response signal of spatial filter support truss.

Table 1. Parameters table of Optomechanical coupling active control system.

\begin{tabular}{ccc}
\hline Parameters & Values & Units \\
\hline $\mathrm{m}$ & 100 & $\mathrm{~kg}$ \\
$\mathrm{c}$ & 120 & $\mathrm{~N} / \mathrm{m} \cdot \mathrm{s}^{-1}$ \\
$\mathrm{k}$ & 40,000 & $\mathrm{~N} / \mathrm{m}$ \\
$\mathrm{K}_{\mathrm{F}}$ & 28 & $\mathrm{~N} / \mathrm{A}$ \\
$\mathrm{L}$ & 0.0053 & $\mathrm{H}$ \\
$\mathrm{R}$ & 8.6 & $\Omega$ \\
\hline
\end{tabular}

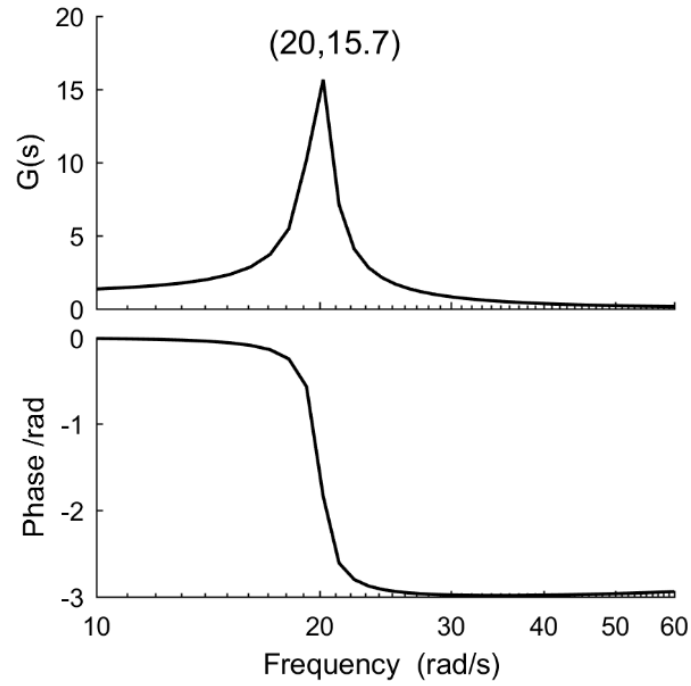

Figure 3. Amplitude frequency and phase frequency response curves of Optomechanical structure.

\subsection{Optomechanical Coupling Active Control System of Spatial Filter}

The spatial filter is a typical system of PW laser facility, as shown in Figure 4. The spectral bandwidth of PW laser is $3 \mathrm{~nm}$, and the central wavelength is $1053 \mathrm{~nm}$. The spatial filter transmission system is composed of $(\mathrm{Obj}$.$) , front lens \left(\mathrm{L}_{i, 1}\right)$, filter pinhole (Filter 
Pinhole), rear lens $\left(\mathrm{L}_{i, 2}\right)$, and image plane (Img.). The object plane reflects the incident light information, which is related to the output light of the rear lens $\left(\mathrm{L}_{i-1,2}\right)$ of the former spatial filter. And the image plane reflects the output light information, which is related to the input light of the front lens $\left(\mathrm{L}_{i+1,1}\right)$ of the latter spatial filter. The spatial filter support truss is simplified as a mass stiffness damping single degree of freedom translational model $\left(\mathrm{m}_{2}-\mathrm{k}_{2}-\mathrm{c}_{2}\right.$ and $\left.\mathrm{m}_{3}-\mathrm{k}_{3}-\mathrm{c}_{3}\right)$, and the active control force $\left(f_{a}\right.$ and $\left.f_{b}\right)$ is applied on the two-stage lens support truss of the spatial filter transmission system to construct the coupling relationship between the Optomechanical structure response and the active control parameters, and the coupling relationship between the Optomechanical structure response and the beam pointing, The relationship between active control parameters and beam pointing is established by two-stage coupling system to improve the output stability of beam propagation spatial filter.

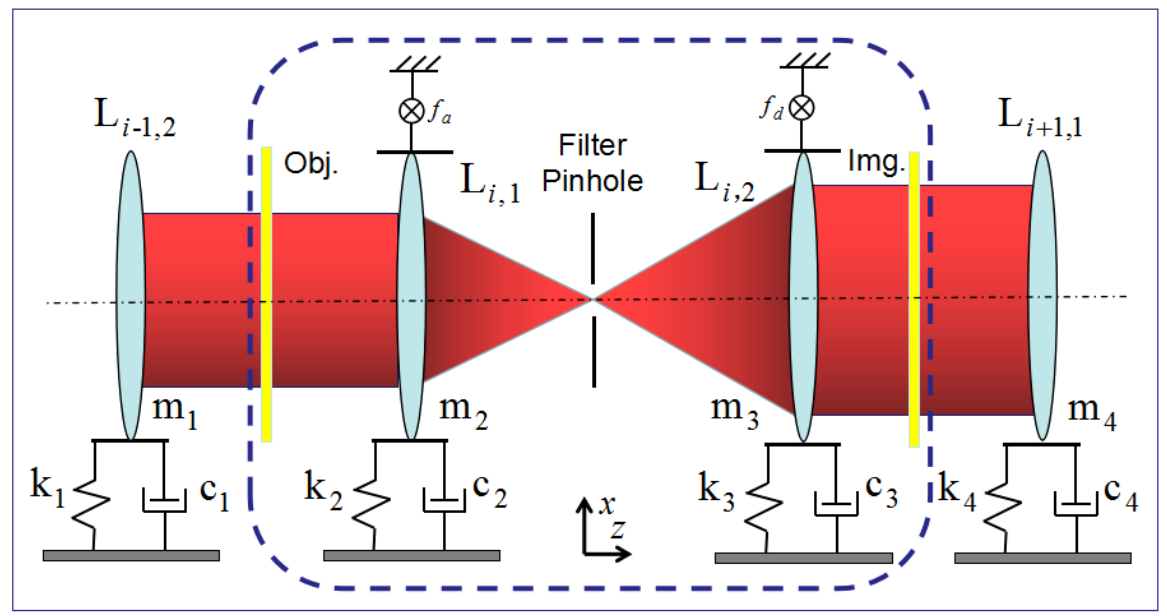

Figure 4. The typical spatial filter system of PW laser facility.

In order to study the beam pointing stability of the Optomechanical coupling system of the spatial filter, the model of the active control system is constructed with the spatial filter whose beam expansion ratio is $m$ in the PW laser device as the object, as shown in Figure 5. The incident beam passes through the thin confocal lens group $L_{1}$ and $L_{2}$ in the spatial filter in turn. According to the modal theory, the $x$ direction first-order translational mode of the support truss is simplified as a single degree of freedom stiffness-mass-damping model. The spatial filter is rigidly connected with the support truss, and the sensor is installed on the support truss of the later lens by using the active feedback control method. The measured offset response is used as the reference of the two support truss systems. By actively controlling the equivalent stiffness and equivalent damping of the two lens support trusses, the difference of the offset response of the two lenses in the $x$ direction is controlled, thus improving the convergence accuracy of the output light angle response.

In this paper, an active control scheme of the Optomechanical system is designed. The scheme is that the two supporting trusses are the same. The supporting trusses of the front and rear lens adopt active control, and the active control law of the two trusses is the same. 


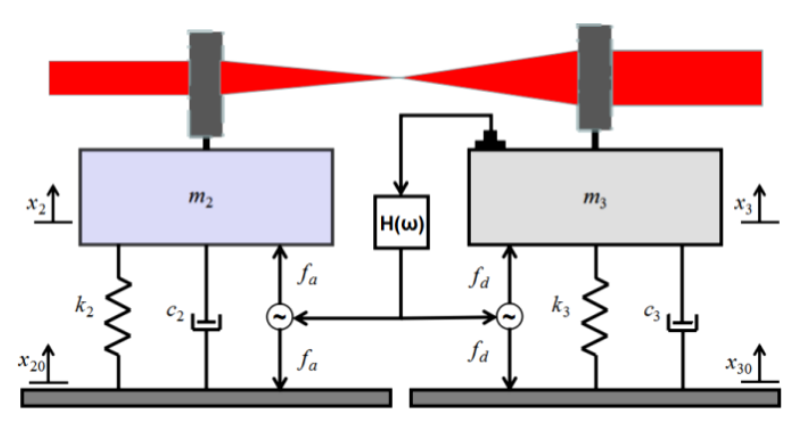

Figure 5. Optomechanical coupling active control system of the spatial filter.

\subsection{Active Controller and Actuator Design}

\subsubsection{PI Controller Design}

The velocity $\dot{x}$ and displacement $x$ of the Optomechanical structure have a great influence on the displacement transfer function of the system, in which $\dot{x}$ can reduce the peak value at the first natural frequency and $x$ can reduce the transfer rate at the low frequency. Using these two quantities as feedback can play the role of active vibration control, and the PI controller is obtained.

$$
h_{c}=g_{I} x+g_{P} \dot{x}
$$

$g_{P}$ is the velocity feedback gain and $g_{I}$ is the displacement feedback gain. The expression of the controller is obtained by Laplace transform. " $s$ " represents the virtual variable, which is the complex frequency domain parameter of the transfer function derived from the Laplace transform.

$$
H_{c}=\left(g_{I}+g_{P} s\right) X
$$

\subsubsection{Actuator Design}

The actuator is an important component in the active control system. Its function is to apply control force to the system according to the control signal to change the system response. Voice coil motor actuator is a new type of actuator based on magnetic force, which has the advantages of fast response, good robustness, and large control force. The voice coil motor actuator can be simplified as the model, shown in Figure 6.

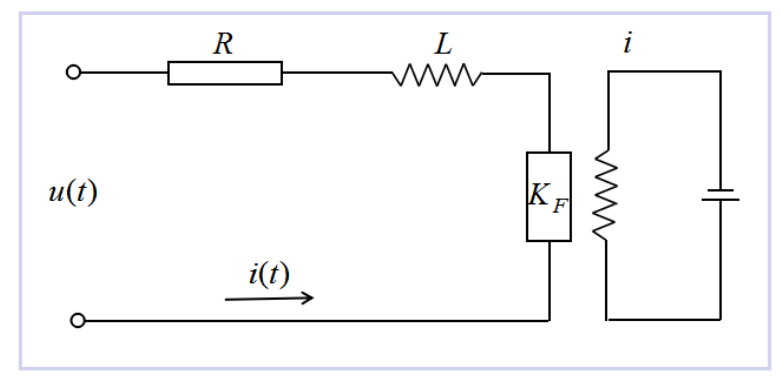

Figure 6. Model diagram of voice coil actuator.

In Figure 6, $i(t)$ is the control current, $R$ is the moving coil resistance, $L$ is the moving coil voltage, $u(t)$ is the control voltage, and $K_{F}$ is the gain. The dynamic analysis of the model of the actuator, shown in Figure 6, shows that the force of the electrified coil in the magnetic field is

$$
F=K_{F} i(t)
$$


According to Kirchhoff voltage law, the electric balance equation of the voice coil motor is obtained.

$$
L \frac{d i(t)}{d t}+R i(t)=u(t)
$$

The AVM50-HF-10 voice coil motor of AKribis company is selected as the actuator for vibration control according to the Optomechanical structural modal parameters of the spatial filter. The parameters of the voice coil motor are $K_{F}=28 \mathrm{~N} / \mathrm{A}, L=0.0053 \mathrm{H}$, $R=8.6 \Omega$, peak thrust $F_{A \max }=140 \mathrm{~N}$, and peak voltage $u_{\max }=43.2 \mathrm{~V}$.

In this paper, when the voice coil motor is used in the actual active control system, the mass of the Optomechanical structure is much larger than that of the motor mover, so the mass of the motor mover can be ignored, and the output force of the motor is used to push the Optomechanical structure, so the transfer function of the voice coil motor can be obtained as follows.

$$
H=\frac{F(s)}{V(s)}=\frac{K_{F}}{L s+R}
$$

The displacement, velocity, and acceleration of the push rod and the Optomechanical structure are equal because the push rod of the actuator is fixedly connected with the Optomechanical structure. This shows that there is a coupling relationship between the actuator and the Optomechanical structure. The active control force of the voice coil motor actuator is

$$
f_{c}=-H \cdot H_{c}=-\frac{K_{F}}{L s+R}\left(g_{I}+g_{P} s\right) X
$$

In this paper, the active actuator uses voice coil motor to provide control force, and adopts a PI control algorithm to control displacement feedback gain and speed feedback gain, which can suppress response amplitude and achieve fast convergence.

\subsection{Dynamics Derivation of Optomechanical Coupling Active Control System}

The motion differential equation of the active control system is as follows

$$
\begin{gathered}
\left\{\begin{array}{l}
m_{2} \ddot{x}_{2}+c_{2} \dot{x}_{2}+k_{2} x_{2}=c_{2} \dot{x}_{20}+k_{2} x_{20}+L^{-1}\left(f_{a}\right) \\
m_{3} \ddot{x}_{3}+c_{3} \dot{x}_{3}+k_{3} x_{3}=c_{3} \dot{x}_{30}+k_{3} x_{30}+L^{-1}\left(f_{d}\right)
\end{array}\right. \\
\left\{\begin{array}{l}
f_{a}=-\frac{K_{F}}{L s+R}\left(g_{I}+g_{P} s\right) X_{2} \\
f_{d}=-\frac{K_{F}}{L s+R}\left(g_{I}+g_{P} s\right) X_{3}
\end{array}\right.
\end{gathered}
$$

Among them, $m_{2}$ and $m_{3}$ are the modal mass, $c_{2}$ and $c_{3}$ are the modal damping, $k_{2}$ and $k_{3}$ are the modal stiffness, $f_{a}$ and $f_{d}$ are the control forces, $x_{20}$ and $x_{30}$ are the foundation disturbance, $x_{2}$ and $x_{3}$ are the lens offset. Since the two supporting trusses are identical, it is noted that $m=m_{2}=m_{3}, c=c_{2}=c_{3}, k=k_{2}=k_{3}$.

The active control force is shown in formula (8), and the pull transformation is carried out by substituting formula (8) into formula (7).

$$
G(s)=G_{2}(s)=G_{3}(s)=\frac{c s+k}{m s^{2}+c s+k-\frac{K_{F}}{L s+R}\left(g_{I}+g_{P} s\right)}
$$

Taking periodic signal as disturbance source, the excitation of two supporting trusses can be expressed as

$$
\left\{\begin{array}{l}
x_{10}=A_{1} \sin \left(\omega_{1} t+\phi_{1}\right) \\
x_{20}=A_{2} \sin \left(\omega_{2} t+\phi_{2}\right)
\end{array}\right.
$$

When the excitation is applied to the Optomechanical coupling active control system, the offset of the two lenses is as follows

$$
\left\{\begin{array}{l}
a_{1}=\frac{c s+k}{m s^{2}+c s+k-\frac{K_{F}}{L+R}\left(g_{I}+g_{P} s\right)} A_{1} \\
a_{2}=\frac{c s+k}{m s^{2}+c s+k-\frac{K_{F}}{L s+R}\left(g_{I}+g_{P} s\right)} A_{2}
\end{array}\right.
$$


$\left(\begin{array}{l}x_{1} \\ \theta_{1}\end{array}\right)$ is the beam incident into the spatial filter, and $\left(\begin{array}{c}x_{2} \\ \theta_{2}\end{array}\right)$ is the beam $\left(\begin{array}{c}x_{1} \\ \theta_{1}\end{array}\right)$ the incident light is transmitted to the next spatial filter through the spatial filter of the Optomechanical coupling active control system, as shown in Figure 7.

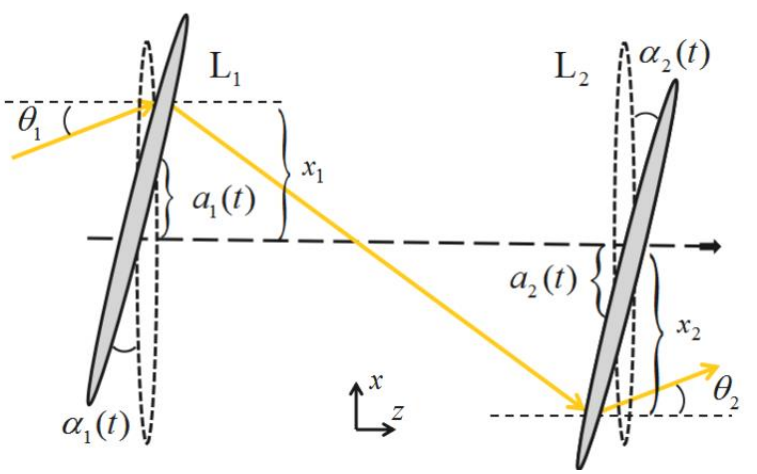

Figure 7. Transmission diagram of active control beam by the spatial filter of PW facility.

According to the beam propagation principle [30], considering one of the beams, the outgoing light can be expressed as

$$
\begin{gathered}
\left(\begin{array}{c}
x_{2} \\
\theta_{2} \\
1 \\
1
\end{array}\right)=\left(\begin{array}{cccc}
1-\frac{l_{12}}{f_{1}} & l_{12} & \frac{l_{12} a_{1}(t)}{f_{1}} & 0 \\
-\left(\frac{1}{f_{1}}+\frac{1}{f_{2}}-\frac{l_{12}}{f_{1} f_{2}}\right) & 1-\frac{l_{12}}{f_{2}} & \frac{a_{1}(t)}{f_{1}}+\frac{a_{2}(t)}{f_{2}}-\frac{l_{12} a_{1}(t)}{f_{1} f_{2}} & 0 \\
0 & 0 & 1 & 0 \\
0 & 0 & 0 & 1
\end{array}\right)\left(\begin{array}{c}
x_{1} \\
\theta_{1} \\
1 \\
1
\end{array}\right) \\
\left\{\begin{array}{l}
l_{12}=f_{1}+f_{2} \\
f_{2}=m f_{1}
\end{array}\right.
\end{gathered}
$$

where $a_{1}(t)$ and $a_{2}(t)$ are the displacement responses of two lenses $L_{1}$ and $L_{2}$ in the $x$ direction, respectively. $f_{1}$ and $f_{2}$ are the focal lengths of lenses $L_{1}$ and $L_{2}$, which $l_{12}$ are the distance from $L_{1}$ and $L_{2}$.

Under ideal conditions, the beam will shoot out parallel to the optical axis; the microvibration of different phases will make the optical axis deviate from the ideal position, and the optical system will be out of alignment; the active control parameters are introduced to modify the beam transmission matrix to reduce the misalignment degree of the optical system and improve the convergence accuracy of the beam direction.

Here, considering the change of the angle of the outgoing light of the lens, the following formulas are obtained.

$$
\theta_{2}=-\frac{1}{m} \theta_{1}-\frac{1}{m f_{1}} a_{1}(t)+\frac{1}{m f_{1}} a_{2}(t)=-\frac{1}{m} \theta_{1}+\frac{1}{m f_{1}}\left[a_{2}(t)-a_{1}(t)\right]
$$

It can be seen from Equation (8) that the angle of light emitted from the transmission system of the spatial filter is the back-and-forth vibration of the angle of $-\frac{1}{m} \theta_{1}$ center. The angle information of outgoing light consists of two parts. The first part is the static value $-\frac{1}{m} \theta_{1}$, which is called the ideal exit angle of $L_{2}$ when the lens is not misaligned; the second part is the dynamic quantity $\frac{1}{m f_{1}}\left[a_{2}(t)-a_{1}(t)\right]$, which is caused by the lens group of spatial optical filter under different phase microvibration and active control, which is the most important part affecting the beam pointing.

Recording the dynamic angular displacement of output light $\Delta \theta_{2}=\frac{1}{m f_{1}}\left[a_{2}(t)-a_{1}(t)\right]$, there are

$$
\Delta \theta_{2}=-\left[\frac{G(s)}{m f_{1}} \sqrt{A_{1}^{2}+A_{2}^{2}-2 A_{1} A_{2} \cos \left[\left(\omega_{1}-\omega_{2}\right) t+\left(\phi_{1}-\phi_{2}\right)\right]}\right] \sin \left(\frac{\omega_{1}+\omega_{2}}{2} t+\frac{\phi_{1}+\phi_{2}}{2}+\varphi\right)
$$


It can be seen from formula (15) that the dynamic angular displacement of the output light has harmonic property, and the amplitude is related to time, which has certain complexity and variability of working conditions. In the analysis, we focus on the amplitude of the dynamic angular displacement of the output light, and use $\Theta$ to represent the peak value of the output light deviation from the ideal angle.

$$
\Theta=\frac{G(s)}{m f_{1}} \sqrt{A_{1}^{2}+A_{2}^{2}-2 A_{1} A_{2} \cos \left[\left(\omega_{1}-\omega_{2}\right) t+\left(\phi_{1}-\phi_{2}\right)\right]}
$$

In the following numerical analysis, take $m f_{1}=f_{2}=16 m$ [31].

Formula (16) shows that the peak value of the output light deviation from the ideal angle is related to the amplitude ratio, frequency difference, and phase difference of the excitation. From the derived formula, it can be found that whether the frequency difference is zero or not determines whether the amplitude of the system response is related to time. When the frequency difference is zero, the peak value of the output light angular response is independent of time, and the beam pointing accuracy depends on the amplitude ratio and phase difference of the excitation. When the frequency difference is not zero, the peak value of the angle response of the output light is time-dependent, and the peak value of the angle response is time-varying. The effects of the amplitude ratio, frequency difference, phase difference, and time on the beam pointing should be considered.

\section{Results and Discussion}

\subsection{The Influence of Excitation on the Peak Value of Output Light Angle Response}

In actual conditions, the different amplitude, frequency, or phase of the two support trusses will affect the vibration response of the Optomechanical structure, and then affect the beam pointing stability of the spatial filter. After the Optomechanical structure design is completed, the influence of different working conditions is studied, and the system is optimized according to the influence degree, and the long-term stability of the beam direction is improved. In this paper, a harmonic signal is used as the source of excitation, and the influence of amplitude ratio $\alpha$, frequency difference $\Delta \omega$, and phase difference $\Delta \phi$ on the directional stability of the spatial filter is analyzed to determine the control parameters of the system. Equation (17) is the expression of amplitude ratio, frequency difference, and phase difference.

$$
\alpha=\frac{A_{2}}{A_{1}}, \Delta \omega=\omega_{1}-\omega_{2}, \Delta \phi=\phi_{1}-\phi_{2}
$$

By substituting Equation (17) into Equation (16), the expression of the peak value of the output light angle response of the spatial filter can be rewritten as

$$
\Theta=\frac{G(s)}{m f_{1}} \sqrt{A_{1}^{2}+\alpha^{2} A_{1}^{2}-2 \alpha A_{1}^{2} \cos [\Delta \omega t+\Delta \phi]}
$$

where, without control, the transfer rate is $G(s)=15.7, m f_{1}=16 m$.

- When the frequency difference is zero $(\Delta \omega=0)$, the peak value of output light angle response is non-time-varying. The influence of excitation amplitude ratio and phase difference on the peak value of output light angle response of the Optomechanical coupling system is analyzed.

It can be seen from Figure 8 that the influence of the excitation amplitude ratio on the peak value of the output light angle response should be analyzed according to the size of the phase difference. When the phase difference is within the range of $[0, \pi / 2)$ and the phase difference is constant, the peak value of the angle response first decreases and then increases with the increase of the amplitude ratio, and there is a minimum point. When the phase difference is zero, and the amplitude ratio is 1 , the peak value of the angle response is 0 . At this time, there is no dynamic drift or jitter in the beam pointing, and 
the beam pointing of the system is only affected by the static deviation. When the phase difference is in the range of $[\pi / 2, \pi]$ and the phase difference is constant, the peak value of angle response increases with the increase of amplitude ratio. When the amplitude ratio is constant, with the increase of the phase difference, the peak value of the output light angle response also increases. The effect of the phase difference on the amplitude of the output light angle response depends on the amplitude ratio.

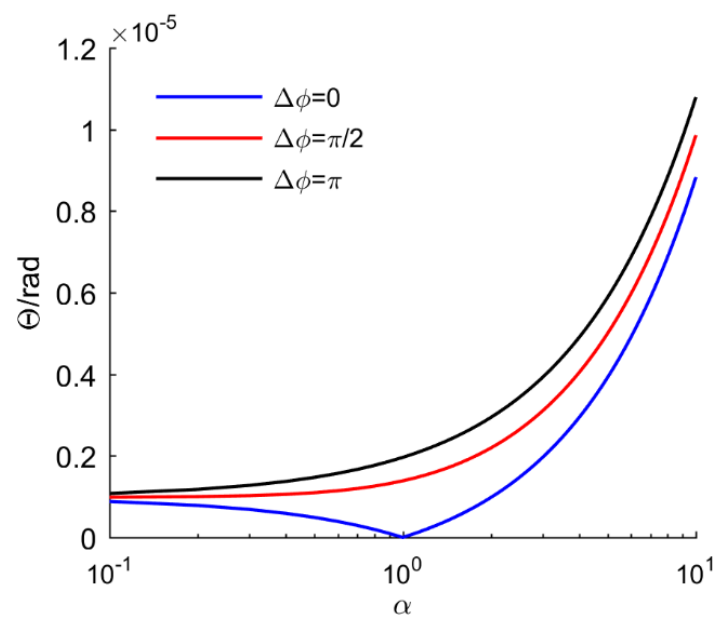

Figure 8. The influence of amplitude ratio and phase difference on the peak value of output light angle response.

- When the frequency difference is zero $(\Delta \omega \neq 0)$, the peak value of output light angle response has time-varying characteristics, and time dimension is a factor that must be considered. The influence of amplitude ratio, frequency difference, and phase difference on the peak value of output light angle response is analyzed.

1. Influence of excitation amplitude ratio on beam pointing accuracy given $\Delta \omega=5$, $\Delta \phi=\pi$;

It can be seen from Figure 9 that the amplitude ratio is positively correlated with the peak value of output light angle response, and the peak value of the angle response increases with the increase of the amplitude ratio.

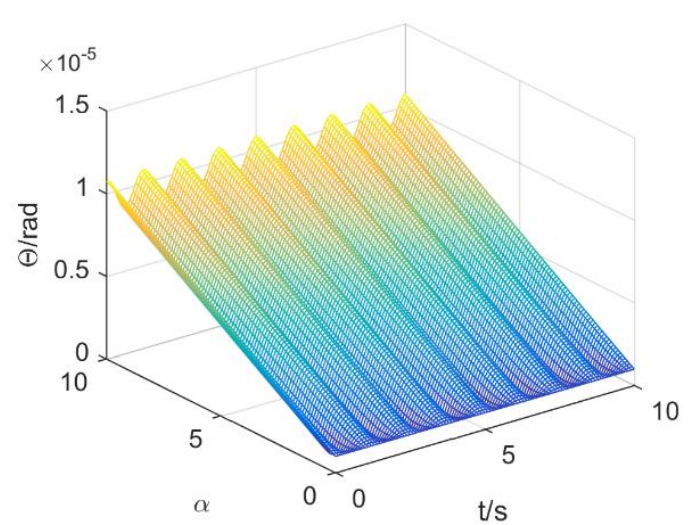

(a)

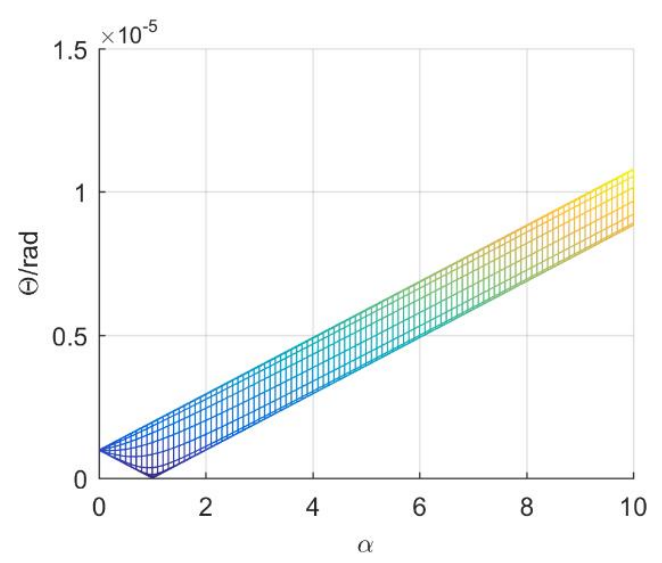

(b)

Figure 9. Given $\Delta \omega=5, \Delta \phi=\pi$, the influence of amplitude ratio on the peak value of output light angle response: (a) The amplitude variation of output light angle response under amplitude ratio and time variables; (b) the relationship between the amplitude ratio and the peak value of the output light angle response. 
2. Influence of excitation frequency difference on beam pointing accuracy given $\alpha=5$, $\Delta \phi=\pi$;

It can be seen from Figure 10 that the change of frequency ratio makes the peak value of angle response change periodically in the range of 3.9-5.8 $\mu \mathrm{rad}$, and the upper and lower limits of the peak value of angular response do not change.

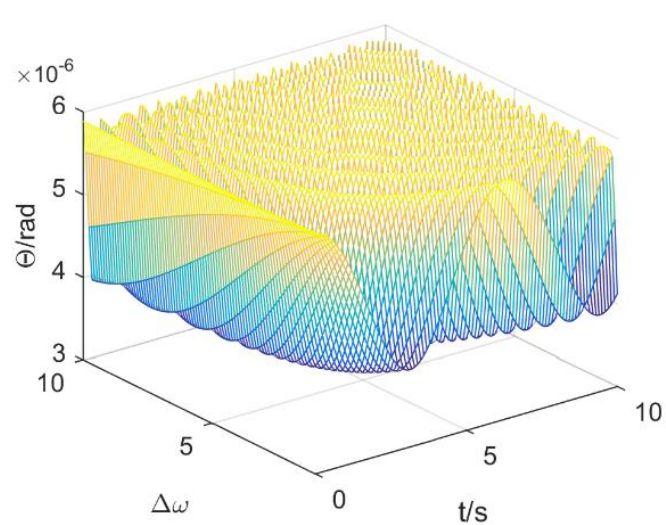

(a)

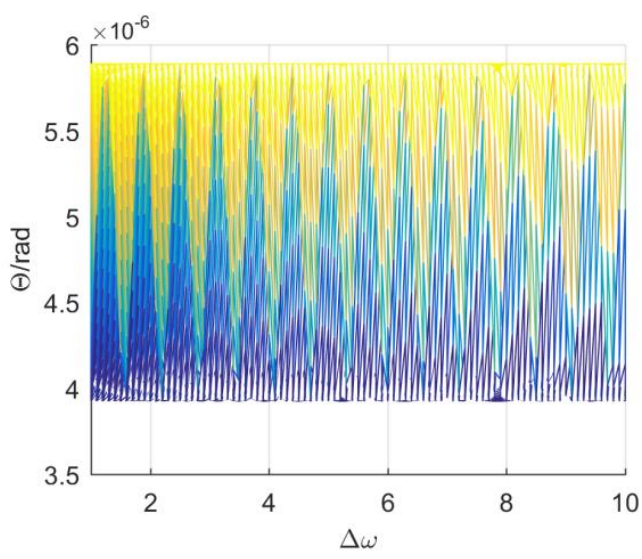

(b)

Figure 10. Given $\alpha=5, \Delta \phi=\pi$, the influence of frequency difference on the peak value of output light angle response: (a) The amplitude variation of output light angle response under frequency difference, and time variable; (b) the amplitude variation of angular response of frequency difference output light.

3. Influence of excitation phase difference on beam pointing accuracy given $\alpha=5$, $\Delta \omega=5$;

It can be seen from Figure 11 that the change of phase difference makes the peak value of angle response change periodically in the range of 3.9-5.8 $\mu \mathrm{rad}$, and the upper and lower limits of the peak value of angular response do not change.

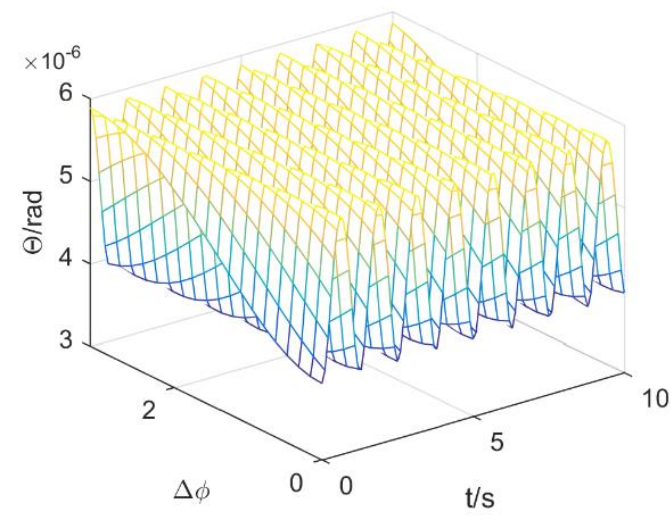

(a)

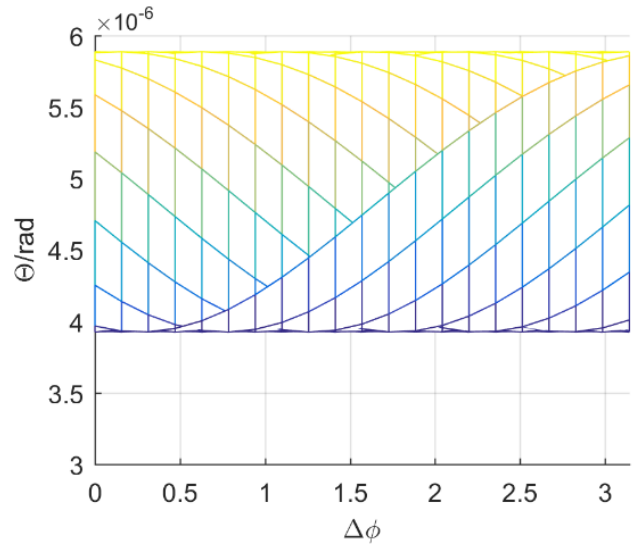

(b)

Figure 11. Given $\alpha=5, \Delta \omega=5$, the influence of phase difference on the peak value of output light angle response: (a) The amplitude variation of output light angle response under phase difference, and time variable; (b) the amplitude variation of angular response of phase difference output light.

From the above analysis, it can be seen that the excitation amplitude ratio has the greatest influence on the peak value of the output light angle response, which is the main factor. When the amplitude ratio is fixed, the frequency ratio and phase difference will make the peak value of the output light angle response change periodically in a fixed 
interval, and the upper and lower limits of this interval are affected by the amplitude ratio. Therefore, to maintain the long-term stability of the beam pointing, the amplitude ratio peak of the output light angle response is controlled within the pointing threshold by the Optomechanical coupling active control system.

\subsection{Active Control Analysis}

\subsubsection{Active Control System Composition and Algorithm Flow}

In the actual working condition, the amplitude ratio of the Optomechanical coupling system of the spatial filter changes, due to the change of environment, and the peak value of the output light angle response will deviate from the pointing threshold. To solve the pointing problem caused by the sudden change of environment, this paper actively adjusts the PI control parameters to control the peak value of the output light angle response within the pointing threshold. MATLAB data processing software is used to study the control effect of active control parameters $g_{P}, g_{I}$ and their joint control on the output light angle response peak value of the spatial filter system.

As shown in Figure 12, the Optomechanical coupling active control system is composed of the Optomechanical system, exciter, sensor, control unit, power amplifier, and actuator. The specific algorithm flow is shown in Figure 13. The sensor detects the peak value of output light angle response in one cycle, and the detection result is compared with the target result. When the detection result is less than or equal to the target result, there is no need to start the actuator. When the detection result is greater than the target result, the deviation between the detection result and the target result is calculated. The controller is started to calculate the control parameter value with zero deviation according to the control algorithm, and then drives the actuator to work. The detection result is compared with the target result again. If the condition is met, the control requirement is met. The cycle control is continued until the result meets the requirement.

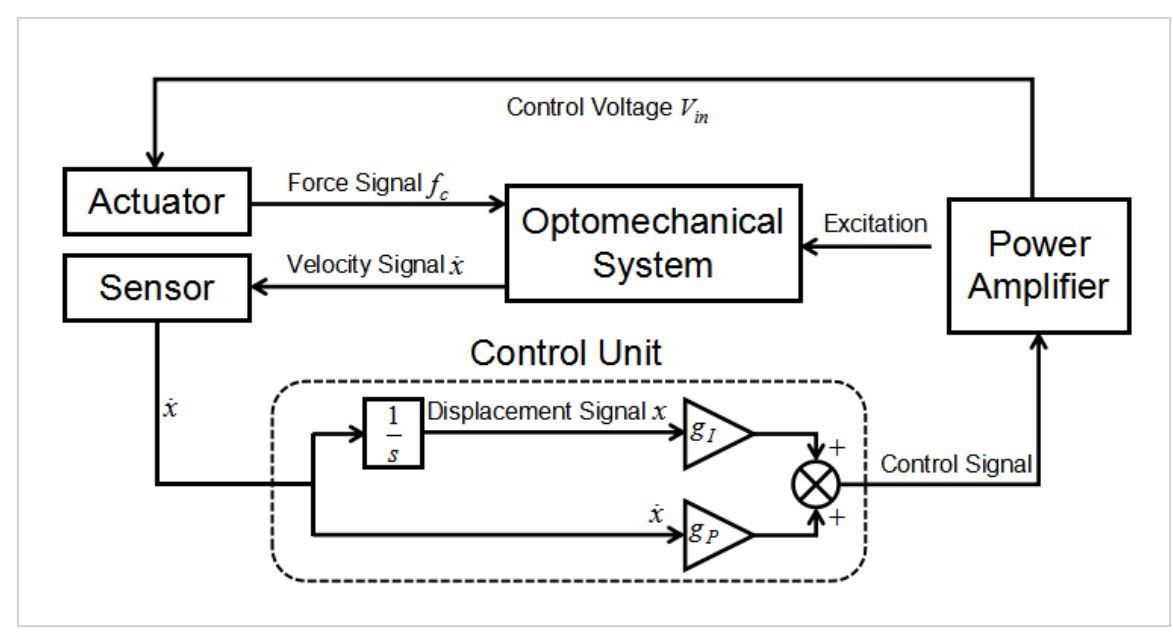

Figure 12. Optomechanical coupling active control system.

According to Figure 12, the closed-loop transfer function can be expressed as

$$
G(s)=\frac{c L s^{2}+(c R+k L) s+k R}{m L s^{3}+(m R+c L) s^{2}+\left(c R+k L-K_{F} g_{P}\right) s+\left(k R-K_{F} g_{I}\right)}
$$

The characteristic equation of the system is

$$
m L s^{3}+(m R+c L) s^{2}+\left(c R+k L-K_{F} g_{P}\right) s+\left(k R-K_{F} g_{I}\right)=0
$$


According to Routh criterion, Routh table is

$$
\begin{array}{c|ccc}
s^{3} & m L & \left(c R+k L-K_{F} g_{P}\right) & 0 \\
s^{2} & (m R+c L) & \left(k R-K_{F} g_{I}\right) & 0 \\
s^{1} & \frac{(m R+c L)\left(c R+k L-K_{F} g_{P}\right)-m L\left(k R-K_{F} g_{I}\right)}{(m R+c L)} & 0 & 0 \\
s^{0} & \left(k R-K_{F} g_{I}\right) & 0 & 0
\end{array}
$$

The sufficient and necessary conditions for the stability of the closed-loop control system are as follows.

(1) The necessary condition for the stability of the system is that the coefficient of the characteristic equation is greater than zero.

$$
\left\{\begin{array}{c}
c R+k L-K_{F} g_{P}>0 \\
k R-K_{F} g_{I}>0
\end{array}\right.
$$

(2) System stability requires that the first column of the Routh table is greater than zero.

$$
\left\{\begin{array}{c}
(m R+c L)\left(c R+k L-K_{F} g_{P}\right)-m L\left(k R-K_{F} g_{I}\right)>0 \\
\left(k R-K_{F} g_{I}\right)>0
\end{array}\right.
$$

Synthesis (1) and (2) the stability condition of the closed-loop system is

$$
\begin{gathered}
0<g_{P}<\min \left\{\frac{c R+k L}{K_{F}}-\frac{m L\left(k R-K_{F} g_{I}\right)}{K_{F}(m R+c L)}, \frac{c R+k L}{K_{F}}\right\} \\
0<g_{I}<\frac{k R}{K_{F}}
\end{gathered}
$$

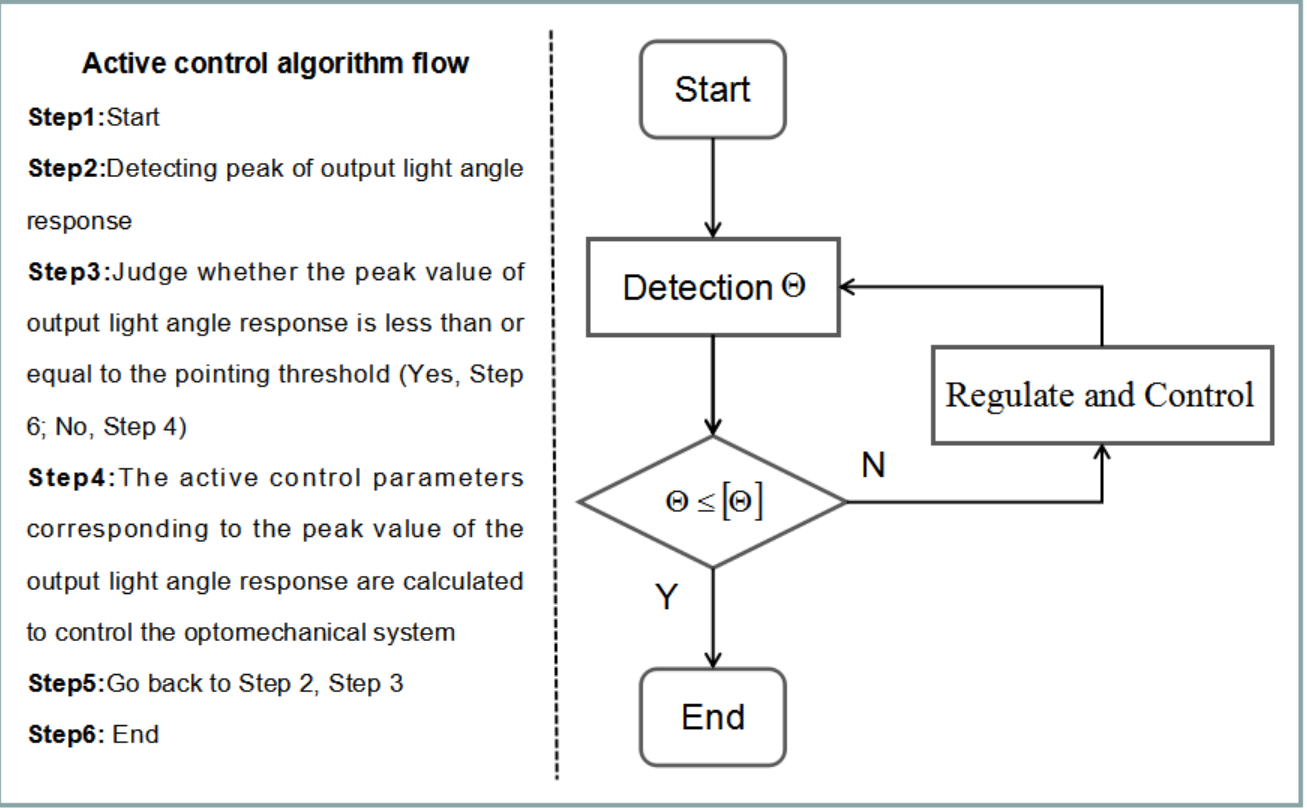

Figure 13. Algorithm flow of the spatial filter Optomechanical coupling active control system.

As can be seen from Figure 13, by detecting the peak value of the output light angle response, the response peak value is compared with the pointing threshold value. If the threshold condition is not met, the output light angle response peak value is detected again by actively controlling the Optomechanical system through the corresponding relationship 
between the output light angle response peak value and the control parameters, until the pointing threshold value is reached.

\subsubsection{Establishment of Control Rules}

Firstly, the relationship between amplitude ratio and control parameters under different excitation frequencies, is obtained through numerical experiments, as shown in Figure 14.

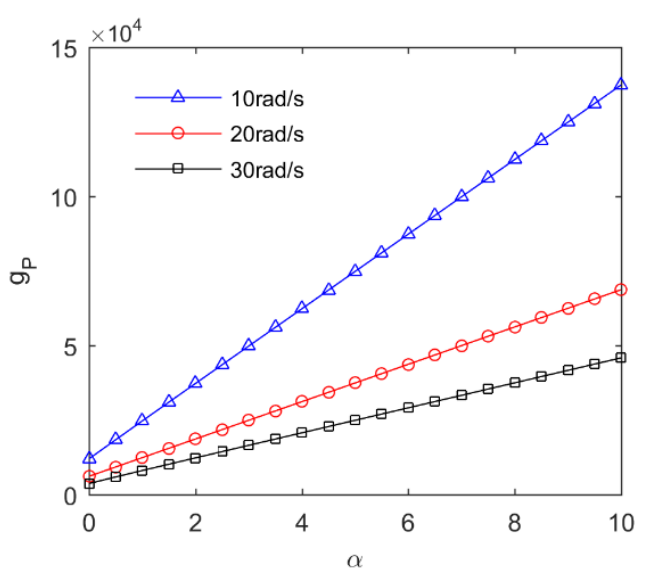

(a)

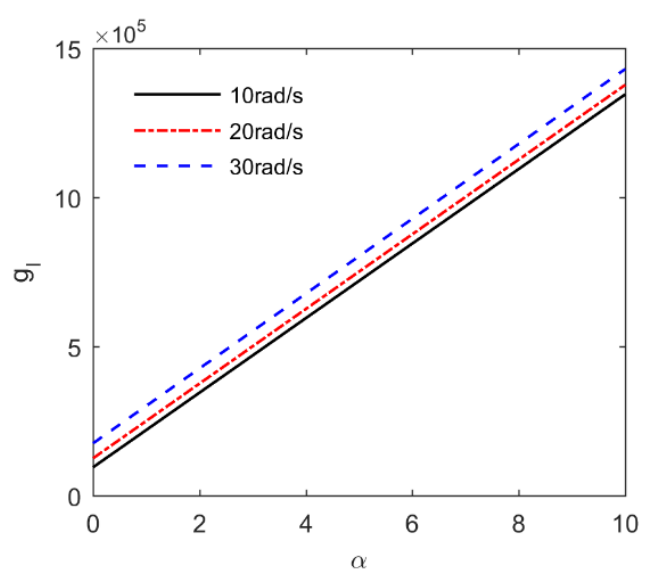

(b)

Figure 14. The relationship between control parameters and amplitude ratio: (a) The relationship between velocity feedback gain and amplitude ratio; (b) the relationship between displacement feedback gain and amplitude ratio. By fitting the experimental data, the relationship between control parameters and the amplitude ratio is obtained as follows.

$$
\begin{gathered}
\left\{\begin{array}{c}
g_{P(10 \mathrm{rad} / \mathrm{s})}=1.253 \times 10^{4} \alpha+1.219 \times 10^{4} \\
g_{P(20 \mathrm{rad} / \mathrm{s})}=6261 \alpha+6141 \\
g_{P(30 \mathrm{rad} / \mathrm{s})}=4204 \alpha+3877
\end{array}\right. \\
\left\{\begin{array}{l}
g_{I(10 \mathrm{rad} / \mathrm{s})}=1.251 \times 10^{5} \alpha+9.505 \times 10^{4} \\
g_{I(20 \mathrm{rad} / \mathrm{s})}=1.252 \times 10^{5} \alpha+1.252 \times 10^{5} \\
g_{I(30 \mathrm{rad} / \mathrm{s})}=1.255 \times 10^{5} \alpha+1.755 \times 10^{5}
\end{array}\right.
\end{gathered}
$$

Secondly, the relationship between the excitation amplitude ratio and the peak value of the output light angle response is analyzed, as shown in Figure 15a.

Thirdly, combined with the previous two steps, the corresponding relationship between the peak value of output light angle response and velocity feedback gain and displacement feedback gain under the excitation of $10 \mathrm{rad} / \mathrm{s}, 20 \mathrm{rad} / \mathrm{s}$, and $30 \mathrm{rad} / \mathrm{s}$ is established, and the accurate active control rules are constructed, as shown in Figure 15b,c.

According to the external excitation frequency and the peak value of output light angle response, the corresponding control parameters in the active control rules are automatically queried. The algorithm calculates the driving actuator to realize the active control of the Optomechanical coupling system. 


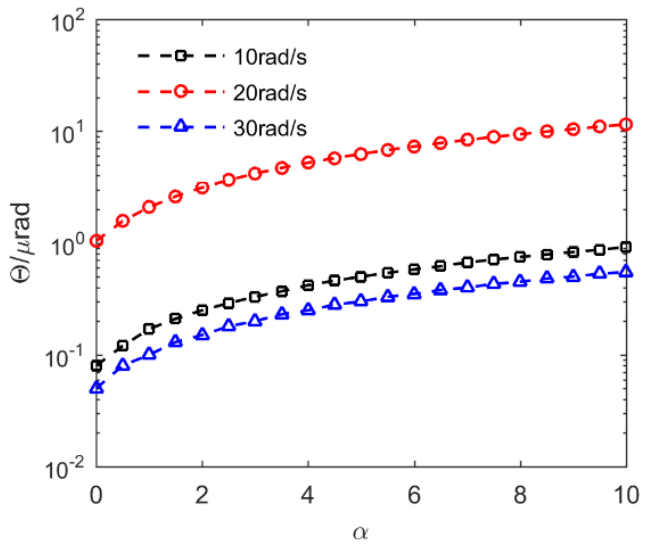

(a)

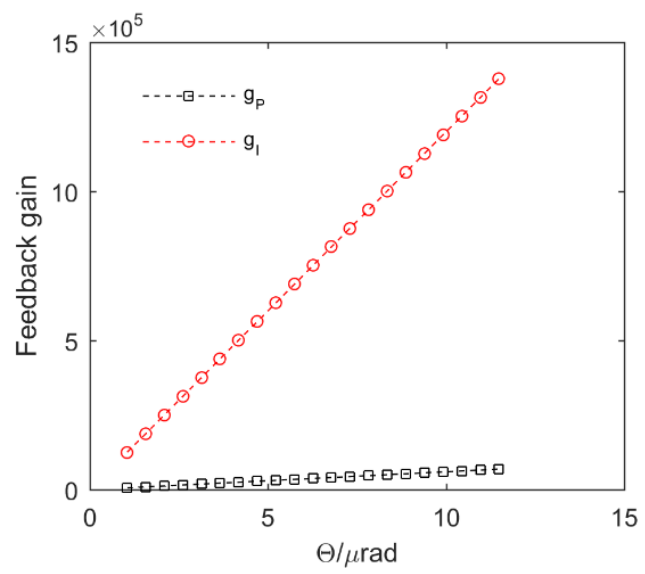

(c)

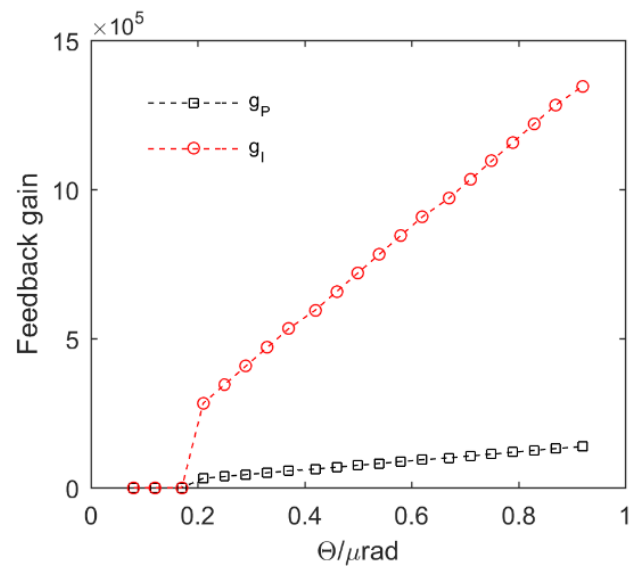

(b)

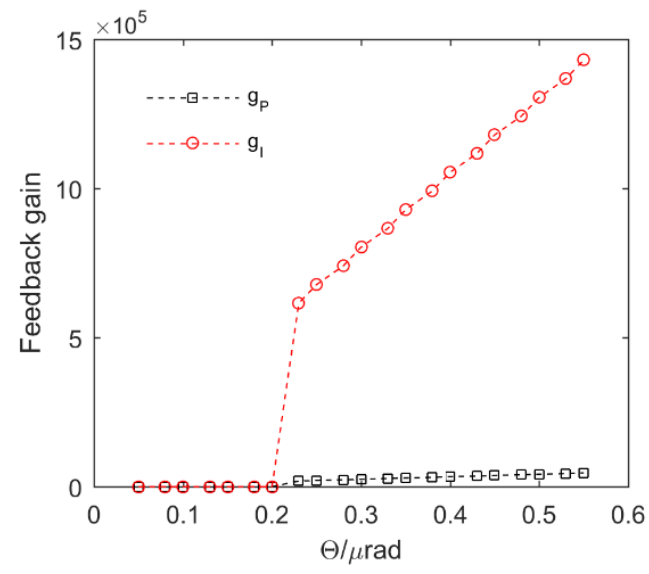

(d)

Figure 15. The corresponding relationship between the parameters of the pointing threshold and the amplitude of the output light angle response when the phase difference $\Delta \varphi=\pi$ is less than $0.2 \mu \mathrm{rad}$ : (a) The corresponding relationship between the ratio of the excitation amplitude and the peak value of the output light angle response when the excitation frequency changes from low frequency to high frequency; (b) the corresponding relationship between the active feedback gain and the peak value of the output light angle response of the Optomechanical system with $10 \mathrm{rad} / \mathrm{s}$ excitation frequency; (c) the corresponding relationship between the active feedback gain and the peak value of the output light angle response of the Optomechanical system with $20 \mathrm{rad} / \mathrm{s}$ excitation frequency; (d) the corresponding relationship between the active feedback gain and the peak value of the output light angle response of the Optomechanical system under the $30 \mathrm{rad} / \mathrm{s}$ excitation frequency.

\subsubsection{Control Effect Verification}

- Two harmonic excitations with excitation frequency of $20 \mathrm{rad} / \mathrm{s}$ and amplitude of $1 \mu \mathrm{m}$ and $5 \mu \mathrm{m}$ act on the Optomechanical coupling active control system.

It can be seen from Figure 16 that in the resonant band, the peak value of the output light angle response reaches the pointing threshold index in the whole frequency domain after the velocity feedback gain $g_{P}$ control is applied. After the displacement feedback gain $g_{I}$ control is applied, the peak value of the output light angle response reaches the pointing threshold index only in the $20 \mathrm{rad} / \mathrm{s}$ resonant band, but it does not reach the pointing threshold index in the low and high frequency bands. The displacement feedback gain requires the range of the excitation frequency band. The joint control of velocity feedback gain $g_{P}$ and displacement feedback gain $g_{I}$ achieves the pointing threshold in the whole frequency domain, but the control effect of ultralow frequency is not as good as that of velocity feedback gain $g_{P}$. 


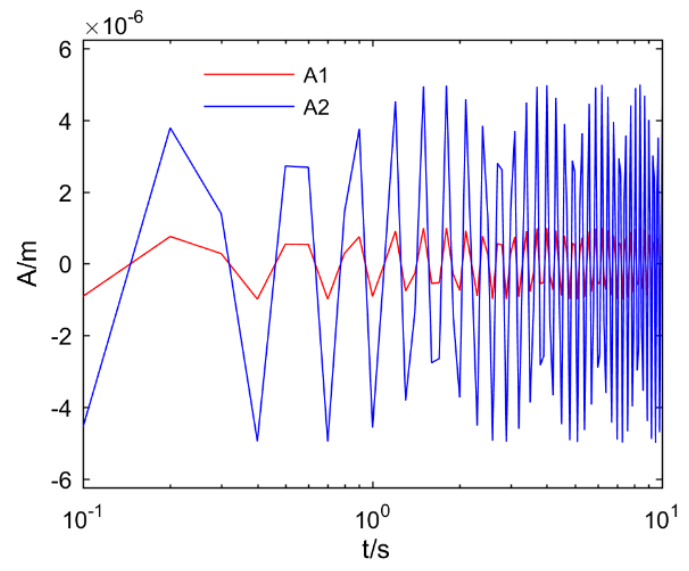

(a)

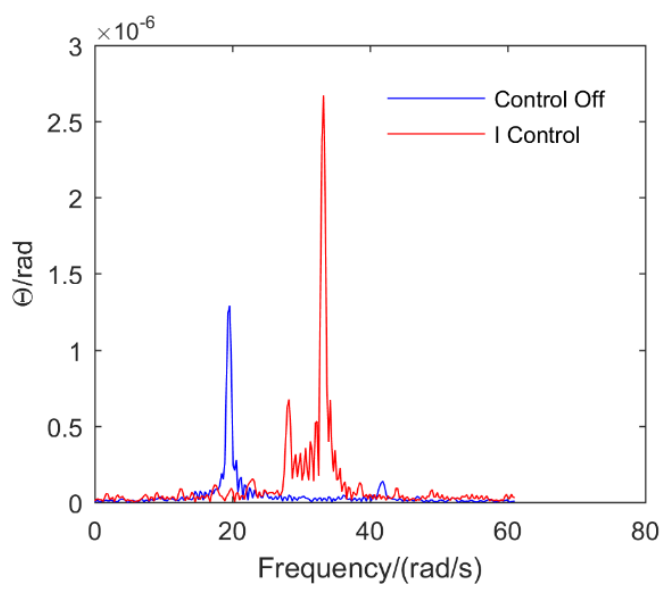

(c)

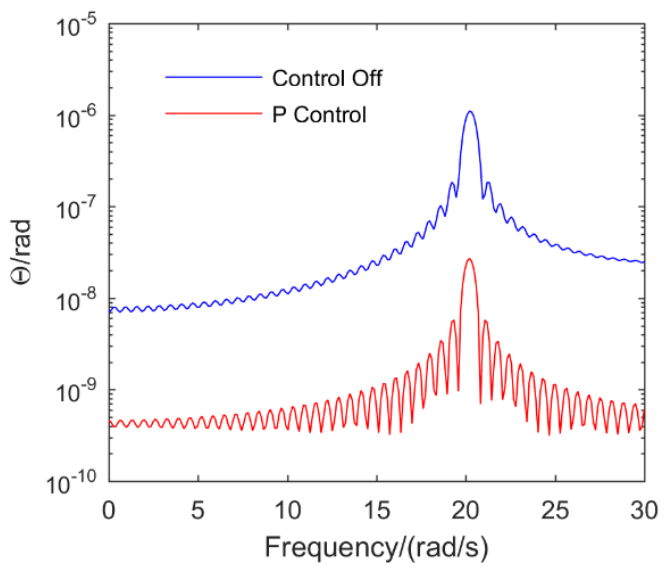

(b)

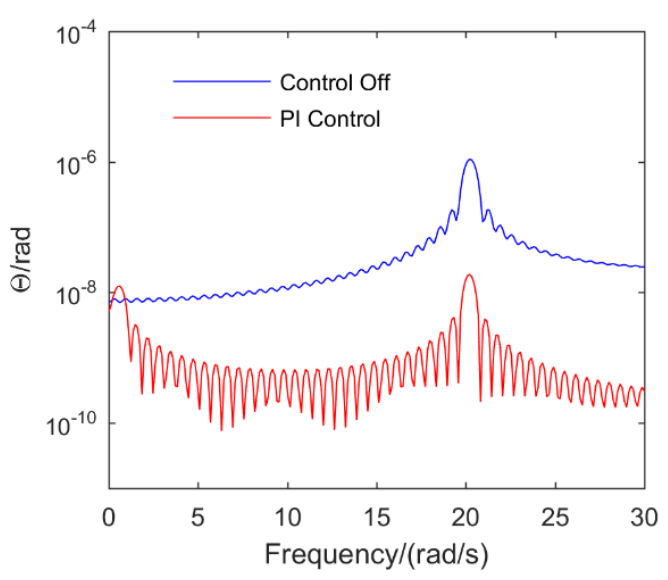

(d)

Figure 16. $20 \mathrm{rad} / \mathrm{s}$ harmonic excitation with and without the control of the spatial filter output light angle response peak comparison: (a) Harmonic excitation signal; (b) velocity feedback gain $g_{P}$ control; (c) displacement feedback gain $g_{I}$ control; (d) velocity feedback gain $g_{P}$ and displacement feedback gain $g_{I}$ joint control.

- Two broadband white noise excitations with power spectral density $10^{-10} \mathrm{~g}^{2} / \mathrm{Hz}$ and $5 \times 10^{-10} \mathrm{~g}^{2} / \mathrm{Hz}$ act on the Optomechanical coupling active control system.

It can be seen from Figure 17a that there is displacement deviation in the 5 times amplitude ratio broadband white noise excitation signal, which causes the optical axis deviation to a certain extent. Figure $17 \mathrm{~b}$ shows the peak value of output light angle response of the velocity feedback gain $g_{P}$ control has reached more than $94 \%$ attenuation near the $20 \mathrm{rad} / \mathrm{s}$ frequency band, and there is not too much control effect in the ultralow frequency part. It can be seen from Figure $17 \mathrm{c}$ that $g_{I}$ can reduce the peak value of angle response near $20 \mathrm{rad} / \mathrm{s}$ frequency band, and the control of low frequency below $10 \mathrm{rad} / \mathrm{s}$ is obvious, but it will lead to high frequency and low peak value. As can be seen from Figure 17d, through the joint control of $g_{I}$ and $g_{P}$, the peak value brought by a under separate control can be reduced. Among all the control methods, the peak attenuation of angular response can reach $98.13 \%$, which is close to two orders of magnitude. When the excitation frequency band is concentrated near the natural frequency, $g_{P}$ control is better. When the excitation frequency band is concentrated in the middle and low frequency region, the control $g_{I}$ is better. When the excitation frequency band is full frequency domain, the joint control of $g_{I}$ and $g_{P}$ is better. 


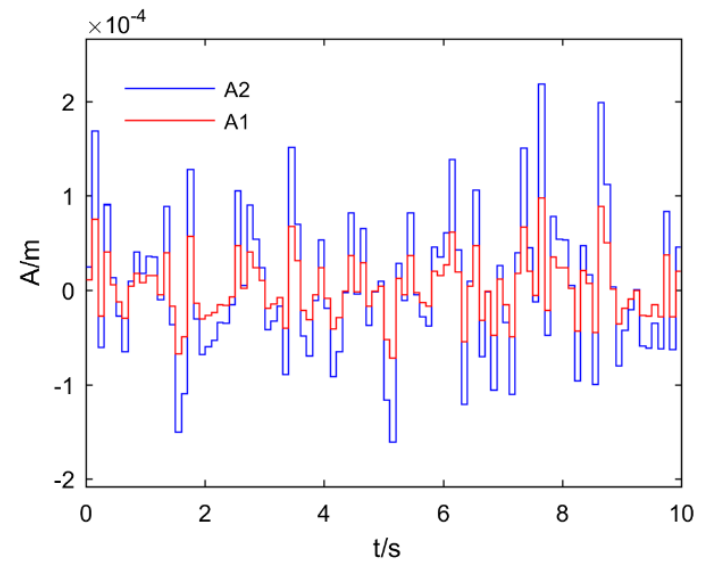

(a)

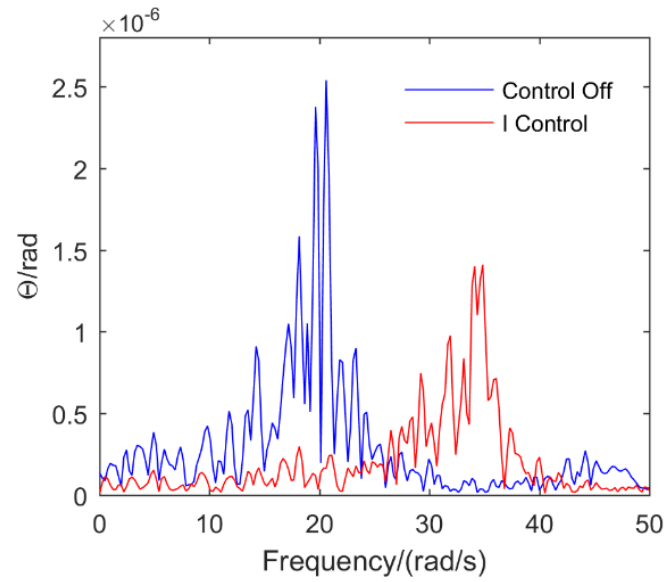

(c)

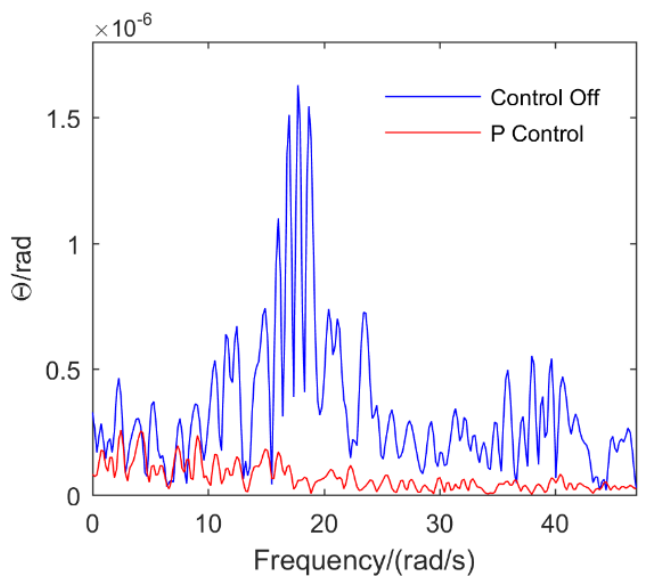

(b)

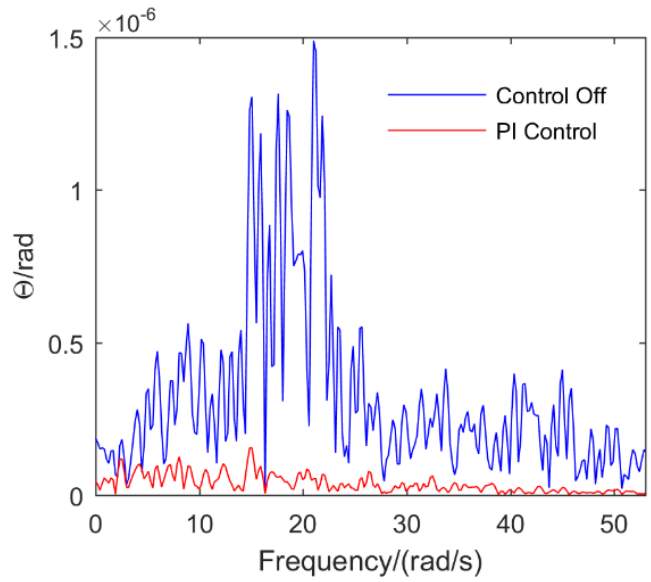

(d)

Figure 17. Comparison of the peak values of output light angle response of the spatial filter under the control and noncontrol of broadband white noise: (a) Displacement excitation signal of broadband white noise; (b) velocity feedback gain $g_{P}$ control; (c) displacement feedback gain $g_{I}$ control; (d) velocity feedback gain $g_{P}$ and displacement feedback gain $g_{I}$ joint control.

Changing the velocity feedback gain $g_{P}$ is equivalent to changing the equivalent damping of the system, and changing the displacement feedback gain $g_{I}$ is equivalent to changing the equivalent stiffness of the system. The control analysis results are consistent with the expectation. Previous studies only analyzed the beam pointing stability by assuming that the structural vibration is known [29], but did not analyze the structural transmission characteristics of the structure under excitation. The results show that this method can effectively control the peak of the output light angle response of the full frequency domain spatial filter and make it fall within the pointing threshold, and the ability to resist environmental changes is significantly enhanced.

The beam stabilization technology is effective for high rate shooting, but it is meaningless for low rate shooting. After the completion of structural design, the space for optimization and improvement is limited. The above two methods may not meet the requirements of a higher beam positioning index. The structure response can be reduced by Optomechanical coupling active control technology to solve the problem of higher beam pointing stability. To achieve better control effect, neural network adaptive online tuning control parameters can be used to intelligently control the structural response in the future, to realize the optimal control under different disturbances.

The most relevant work has only considered the influence of vibration factors on beam pointing, and has not studied the control of the Optomechanical system. Other contemporary methods are studied from the beam stabilization technology, this part of the 
influence on the beam pointing is very small, and the main influence factor of the beam misalignment is caused by the vibration of the Optomechanical structure, accounting for more than $60 \%$ [23], so controlling the response of the Optomechanical structure is more effective to improve the beam pointing.

\section{Conclusions}

In this paper, the theory of Optomechanical coupling vibration active control is proposed. Through the first coupling between active control parameters and structural response and the second coupling between structural response and beam pointing, the active control parameters are introduced into the beam transmission matrix of the Optomechanical coupling system, and the point-to-point link between active control parameters and beam pointing is realized. An active control scheme with different excitation is implemented to solve the problem of output light angle misalignment caused by the deviation of the amplitude response of the spatial filter confocal lens under different microvibration excitation. When the microvibration phase of the confocal lens is opposite, the output light angle of the spatial filter transmission system oscillates back and forth with $-\frac{1}{m} \theta_{1}$ as the center angle. Under the active control, the maximum amplitude of the output light angle oscillation deviation is reduced by $98.13 \%$, and the attenuation is close to two orders of magnitude. To a certain extent, the convergence speed of the beam pointing is improved, and the dual control of the peak value of the output light angle response and the convergence speed is realized. The results show that the proposed method can effectively control the peak value of the output light angle response of the full frequency domain spatial filter and make it fall within the pointing threshold. The control analysis results are consistent with the expectation. The research results can guide cascaded multi spatial filters and other similar systems.

Author Contributions: Conceptualization, W.C. and J.Z. (Jianqiang Zhu); methodology, W.C.; software, W.C.; validation, Z.L., Z.J., Q.F. and J.Z. (Junyong Zhang).; formal analysis, W.C.; investigation, W.C.; resources, J.Z. (Jianqiang Zhu); data curation, W.C.; writing-original draft preparation, W.C.; writing-review and editing, W.C.; visualization, W.C.; supervision, W.L. and Z.D.; project administration, W.C.; funding acquisition, W.C. All authors have read and agreed to the published version of the manuscript.

Funding: This research was funded by the International Partnership Program of Chinese Academy of Sciences (No.181231KYSB20170022), and the Strategie Priority Research Program of Chinese Academy of Sciences (No.XDA25020304).

Institutional Review Board Statement: Not applicable.

Informed Consent Statement: Not applicable.

Data Availability Statement: Data are available from the corresponding author upon reasonable request.

Conflicts of Interest: The authors declare no conflict of interest. The funders had no role in the design of the study; in the collection, analyses, or interpretation of data; in the writing of the manuscript, or in the decision to publish the results.

\section{References}

1. Speck, D.; Bliss, E.; Glaze, J.; Herris, J.; Holloway, F.; Hunt, J.; Johnson, B.; Kuizenga, D.; Ozarski, R.; Patton, H.; et al. The Shiva laser-fusion facility. IEEE J. Quantum Electron. 1981, 17, 1599-1619. [CrossRef]

2. Burkhart, S.C.; Bliss, E.; Di Nicola, P.; Kalantar, D.; Lowe-Webb, R.; McCarville, T.; Nelson, D.; Salmon, T.; Schindler, T.; Villanueva, J.; et al. National Ignition Facility system alignment. Appl. Opt. 2011, 50, 1136-1157. [CrossRef]

3. Hooker, C.J.; Collier, J.L.; Chekhlov, O.; Clarke, R.; Divall, E.; Ertel, K.; Fell, B.; Foster, P.; Hancock, S.; Langley, A.; et al. The Astra Gemini project-A dual-beam petawatt Ti: Sapphire laser system. J. Phys. IV Proc. 2006, 133, 673-677.

4. Cavailler, C.; Noel, A.; Nicola, J.M.D. LIL and LMJ laser facility status. Proc. SPIE 2005, 5580, 443-454.

5. Zhu, J.Q. Shenguang-II high power laser facility. Bull. Chin. Acad. Sci. 2005, 20, 42-44,85.

6. Zhao, D.F.; Dai, Y.P. Error analysis for the optical system of target area on high power laser facility. Chin. J. Lasers 2004, 31, 1425-1428. 
7. Zhu, J.; Zhu, J.; Li, X.; Zhu, B.; Ma, W.; Lu, X.; Fan, W.; Liu, Z.; Zhou, S.; Xu, G.; et al. Status and development of high-power laser facilities at the NLHPLP. High Power Laser Sci. Eng. 2018, 4, e55. [CrossRef]

8. Zhu, J.Q. Review of special issue on high power facility and technical development at the NLHPLP. High Power Laser Sci. Eng. 2019, 1, 94-95. [CrossRef]

9. Zhang, J.; Zhang, S.; Lin, D.; Fu, X.; Chen, L.; Zhou, Y. Study on Control Strategy of Structure Stability for the Shenguang-III Laser Facility. Fusion Eng. Des. 2017, 120, 27-33. [CrossRef]

10. Danson, C.N.; Haefner, C.; Bromage, J.; Butcher, T.; Chanteloup, J.C.; Chowdhury, E.A.; Galvanauskas, A.; Gizzi, L.A.; Hein, J.; Hillier, D.I.; et al. Petawatt and exawatt class lasers worldwide. High Power Laser Sci. Eng. 2019, 7, 1-54. [CrossRef]

11. Liang, X.; Xie, X.; Kang, J.; Yang, Q.; Wei, H.; Sun, M.; Zhu, J. Design and experimental demonstration of a high conversion efficiency OPCPA pre-amplifier for petawatt laser facility. High Power Laser Sci. Eng. 2018, 4, 60-68. [CrossRef]

12. Zhu, J.; Xie, X.; Sun, M.; Kang, J.; Yang, Q.; Guo, A.; Zhu, H.; Zhu, P.; Gao, Q.; Liang, X.; et al. Analysis and construction status of SG-II 5PW laser facility. High Power Laser Sci. Eng. 2018, 2, e29. [CrossRef]

13. Homoelle, D.; Baker, K.L.; Patel, P.K.; Utterback, E.; Rushford, M.C.; Siders, C.W.; Barty, C.P. Interferometric adaptive optics for high-power laser beam correction in fast ignition experiments. J. Phys. Conf. Ser. 2010, 244, 032007. [CrossRef]

14. Mahdieh, M.H.; Shirmahi, M.; Alavi-Nejad, M. Beam quality in unstable optical resonators and the effects of misalignment. Workshop Laser Appl. Eur. 2006, 6157, X1570.

15. Gao, Y.Q.; Zhu, B.Q.; Liu, D.Z.; Lin, Z.Q. Influences of the alignment and misalignment spatial filters on the beam quality in high power laser systems. J. Opt. 2010, 12, 095704. [CrossRef]

16. Wang, J.; Huang, L.; Hou, L.; He, G.; Song, Q.; Huang, H. Study of active beam steering system with a simple method. Chin. Opt. Lett. 2014, 12, 55-59.

17. Chang, H.; Ge, W.Q.; Wang, H.C.; Yuan, H.; Fan, Z.W. Laser Beam Pointing Stabilization Control through Disturbance Classification. Sensors 2021, 21, 1946. [CrossRef]

18. Okamoto, H.; Watanabe, T.; Ohta, R.; Onomitsu, K.; Gotoh, H.; Sogawa, T.; Yamaguchi, H. Cavity-less on-chip optomechanics using excitonic transitions in semiconductor heterostructures. Nat. Commun. 2015, 6, 8478. [CrossRef] [PubMed]

19. Jiang, W.H. Adaptive optics and active optics. Physics 1997, 26, 73-79.

20. Babcock, H.W. The possibility of compensating astronomical seeing. Publ. Astron. Soc. Pac. 1953, 65, 229-236. [CrossRef]

21. McMickell, M.B.; Kreider, T.; Hansen, E.; Davis, T.; Gonzalez, M. Optical payload isolation using the miniature vibration isolation system (mvis-ii). Ind. Commer. Appl. Smart Struct. Technol. 2007, 6527, 52703.

22. Lee, D.O.; Yoon, J.S.; Han, J.H. Integrated framework for fitter analysis combining disturbance, structure, vibration isolator and optical model. Act. Passiv. Smart Struct. Integr. Syst. 2012, 8341, 834126.

23. Sommer, S.C.; Bliss, E.S. NIF Systems Engineering Systems Control Engineer. In Chapter 11 of Beam Positioning: NIF Laser System Performance Ratings; UCRL-ID-131115; LLNL: Livermore, CA, USA, 1998.

24. Swensen, E.A.; Barron, A.A.; Farrar, C.R.; Cornwell, P. The 15th International Modal Analysis Conference. Proc. SPIE 1996, 3089, 1172.

25. Zeng, T.Y. Stability Analysis for Some Key Elements and Systems on High Power Laser Driver in ICF. Ph.D. Thesis, Shanghai Institute of Optics and Fine Mechanics, Chinese Academy of Sciences, Shanghai, China, 2007.

26. Yang, H.W.; Tao, W.; Zhang, Z.Q.; Zhao, S.W.; Yin, X.Q.; Zhao, H. Reduction of the Influence of Laser Beam Directional Dithering in a Laser Triangulation Displacement Probe. Sensors 2017, 17, 1126. [CrossRef] [PubMed]

27. Qin, F.; Zhang, D.; Xing, D.; Xu, D.; Li, J. Laser Beam Pointing Control with Piezoelectric Actuator Model Learning. IEEE Trans. Syst. Man Cybern. Syst. 2020, 50, 1024-1034. [CrossRef]

28. Liu, F.; Zhu, J.; Xu, J.; Shan, Q.; Xiao, K.; Zhang, X. Micro-vibrating spatial filters-induced beam positioning stability in laser system. Chin. Opt. Lett. 2012, 10, 04402.

29. Liu, F.; Xu, J.; Zhang, Y.; Sun, M.Y.; Zhu, J.Q. Beam pointing stability analysis caused by micro vibration of optical devices. Acta Opt. Sin. 2011, 31, 1120001.

30. Lu, B.D. Laser Optics: Laser Beam Transformation and Beam Quality Control; Sichuan University Press: Sichuan, China, 1992; pp. 339-346.

31. Liu, F. Beam Pointing Stability Analysis and Improvement of High Power Laser Facility. Ph.D. Thesis, Shanghai Institute of Optics and Precision Mechanics, Chinese Academy of Sciences, Shanghai, China, 2012; pp. 46-52. 